Sharif University of Technology
Scientia Iranica
Special Issue on: Socio-Cognitive Engineering
SCIENTIA
I RAN I CA

\title{
Increasing stability in model-mediated teleoperation approach by reducing model jump effect
}

\author{
B. Yazdankhoo and B. Beigzadeh* \\ School of Mechanical Engineering, Iran University of Science and Technology, Tehran, P.O. Box 16765163, Iran.
}

Received 8 March 2017; received in revised form 8 October 2017; accepted 18 December 2017

\section{KEYWORDS}

Predictive control; Model-mediated teleoperation; Transparency; Model jump; Decoupling method; Sliding mode control.

\begin{abstract}
Model-mediated teleoperation is a predictive control approach to controlling haptic teleoperation systems whereby the environment force is virtually located on master side in order to increase the stability and transparency of the system. This promising approach, however, resulted in new challenges. One pivotal challenge is the model jump effect, which stems from the delay in correct creation of the virtual environment. Previous works endeavored to reduce this effect; however, they either led to transparency decrease or assumed simplified environment models. This paper proposed a control approach for this aim based on the idea of decoupling. This means that when a new environment has been identified, the operation is interrupted and no signal is transmitted between master and slave sides. During this time, both sides are controlled by their own sliding mode controllers until the system reaches stability. The main advantage of this method is its independence of environment type, which makes it usable for different kinds of applications. To verify the effectiveness of the proposed approach, simulation tests are conducted. The results show that the system is stable in interaction with hard and soft environments in the presence of large time delays in communication channels.
\end{abstract}

(C) 2019 Sharif University of Technology. All rights reserved.

\section{Introduction}

Design and control of haptic teleoperation systems has gained many researchers' attention over the past decades. Conventional control methods have long been utilized in teleoperation systems as control strategies by adopting classical control architectures, such as position-position, force-position, or 4C (fourchannel) [1]. All these architectures are characterized with the method of sending the intended signals (position and/or force) through communication channels to the other side, where the delayed signals are employed

\footnotetext{
*. Corresponding author. Tel.: +9821 rra240094; Fax: +9821 73021587

E-mail addresses: b_yazdankhoo@mecheng.iust.ac.ir (B. Yazdankhoo); b_beigzadeh@iust.ac.ir (B. Beigzadeh)
}

doi: $10.24200 /$ sci. 2017.20007 to design the desired controller, in common. In position-position architecture, position signals are sent from each side to the other side. This architecture was used in [2], for instance, in order to design a teleoperation system. Another prevalent type of architecture, which resembles $4 \mathrm{C}$, is the one that sends position and force signals from master to slave side; yet, it only sends force signal from slave to master side. Many teleoperation systems have been designed based on this architecture such as [3,4]. These control architectures, however, fail to provide high transparency. This happens because a compromise should always be considered between the two conflicting objectives: transparency and stability [5]. It should be noted that, herein, transparency means the quality of position and force tracking of the system. In other words, the extent to which the slave and master robots are able to replicate the position of the master and the environment force, respectively, is called transparency. Although 
quantitative definitions were also presented [1], we stick to the qualitative definition of transparency herein, since it is sufficient to describe the results of the proposed method.

To conquer the deficiency mentioned in the previous paragraph, predictive control approaches were introduced in teleoperation systems, primarily by researches such as [6] which attempted to exploit online estimated parameters instead of actual transmitted ones. The main advantage of these approaches is that they are able to circumvent the time delay existing in the communication channels, thus making the system more transparent and stable. Various predictive control methods have been proposed in the literature up to now [7], in which the researches have been focused on predicting the environment force on the master side, which is typically called model-mediated teleoperation. In other words, according to this approach, the environment impedance is estimated on the slave side and, then, transmitted to the master side, where the force is locally recreated based on the position and velocity of the master robot. A general scheme of this approach is depicted in Figure 1.

In spite of providing high transparency, the model-mediated approach creates some new challenges. One of the main challenges is that when the slave robot encounters a new environment, obtaining the true environment impedance may take some time, and also rendering them to the master side is delayed due to the nature of the communication channels. During this period, the master side is not aware of the true environment parameters; thus, the local force cannot be created correctly. This time interval, which is called the period of model mismatch, puts the system in an unstable situation which needs to be suitably controlled [8].

Stability of model-mediated teleoperation has been addressed by some researches in recent years. Some of the proposed approaches provide stability at the expense of transparency, such as [9], which proposes the idea of displacement of the local virtual

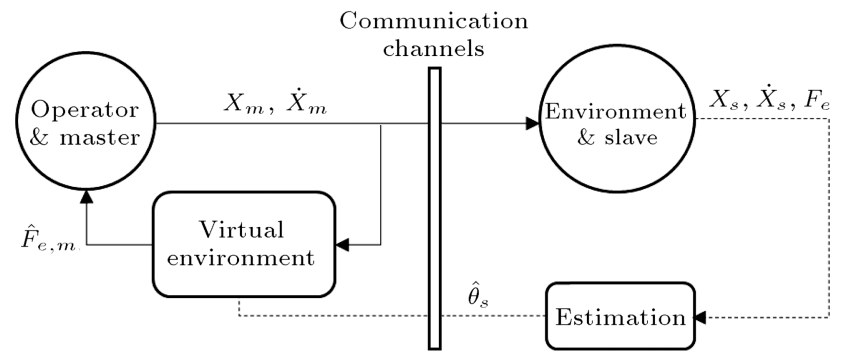

Figure 1. General scheme of model-mediated teleoperation approach. $x, F_{e}$, and $\hat{F}_{e, m}$ represent position, environment force, and estimated environment force, respectively. $\hat{\theta}_{s}$ is also the estimated environment parameters. Subscripts $m$ and $s$ denote master and slave, respectively. environment in the direction of the movement of the master robot to stabilize the system when the first contact on the slave side occurs. Passivity-based model updating is another technique which is adopted in [10] by inducing an additional damping factor in the virtual environment when a change in model happens. This may also lead to a decrease in transparency depending on the type of model update. The other proposed approaches, however, have reached the ultimate goal of stabilizing the system through the assumption of a simple environment model $[11,12]$. As a consequence, presenting a control method that can conserve system stability and transparency together, regardless of the environment type, seems necessary.

In this paper, a control strategy for the period of model mismatch (or equivalently transition state) is incorporated so that the system stability can be preserved even for large amounts of time delay. The strategy is based on the idea of full decoupling whereby master and slave sides are decoupled when a new environment appears. In that period, no signal is transmitted between master and slave sides, and both sides are separately controlled by their own controllers. These controllers are also independent of environment type; therefore, the system is able to interact with both hard and soft environments.

The rest of this paper is organized as follows. Section 2 describes the dynamic modeling of the system. Section 3 is dedicated to explaining the estimation algorithms used to estimate the impedance for both hard and soft environments. Section 4 describes the controllers designed for the different states of the system and presents discussion on the overall stability of the system. Finally, Section 5 provides the simulation results, while conclusions and suggestions for future researches are included in Section 6 .

\section{System modeling}

\subsection{Master side modeling}

The dynamics of the master robot is considered as a one-degree-of-freedom, linear second-order system in time domain, as shown in Eq. (1):

$$
m_{m} \ddot{x}_{m}+b_{m} \dot{x}_{m}+k_{m} x_{m}=F_{h}^{*}+U_{m}
$$

where $m, b$, and $k$ denote the mass, damping coefficient, and stiffness, respectively. $F_{h}^{*}$ is the interaction force between the operator and the master robot, and $U$ is the control input. $x$ represents the position of the robot, and subscript $m$ denotes the master robot.

For simulation purposes, it is necessary to obtain a model for human or operator hand. Several models have been proposed for representing the dynamic behavior of human hand during interaction with teleoperation systems. The first and possibly most well-known 
model is the linear second-order one proposed in [13]. However, the authors of [14] proved that a suitable model should be of relative order one. Hence, they derived a two-parameter model based on experimental data that can be written as follows:

$$
b_{a} \dot{x}_{m}+k_{a} x_{m}=F_{h}-F_{h}^{*},
$$

where $b_{a}$ and $k_{a}$ represent the damping and stiffness coefficients of hand, respectively, and $F_{h}$ is the human exogenous force. It should be pointed out that, in Eq. (2), it is assumed that the operator continuously and completely grasps the end effector of the master robot, such that the position of his/her hand is always equal to the position of the robot.

By combining Eqs. (1) and (2), the dynamics of the master side can be obtained, which is given by:

$$
m_{m} \ddot{x}_{m}+b_{t, m} \dot{x}_{m}+k_{t, m} x_{m}=F_{h}+U_{m},
$$

where $b_{t, m} \triangleq b_{m}+b_{a}$ and $k_{t, m} \triangleq k_{m}+k_{a}$ are assumed.

\subsection{Slave side modeling}

The dynamics of the slave robot is considered as a onedegree-of-freedom, linear second-order system in time domain, as indicated in Eq. (4):

$$
m_{s} \ddot{x}_{s}+b_{s} \dot{x}_{s}+k_{s} x_{s}=U_{s}-F_{e},
$$

where $m, b$, and $k$ denote the mass, damping coefficient, and stiffness, respectively; $F_{e}$ is the environment force and $U$ is the control input; $x$ specifies the position of the robot; and subscript $s$ denotes the slave robot.

The environment force can be represented by the augmented Kelvin-Voigt (KV) model for hard environments and the augmented Hunt-Crossley (HC) model for soft environments, which are given by Eqs. (5) and (6), respectively:

$$
\begin{aligned}
& F_{e}= \begin{cases}K_{\mathrm{KV}} \delta x_{s}+B_{\mathrm{KV}} \dot{x}_{s}, & \delta x_{s}>0 \wedge \dot{x}_{s} \geq 0 \\
K_{\mathrm{KV}} \delta x_{s}, & \delta x_{s} \geq 0 \wedge \dot{x}_{s}<0 \\
0, & \text { else. }\end{cases} \\
& F_{e}= \begin{cases}K_{\mathrm{HC}} \delta x_{s}{ }^{n}+B_{\mathrm{HC}} \delta x_{s}{ }^{n} \dot{x}_{s}, & \delta x_{s} \geq 0 \wedge \dot{x}_{s} \geq 0 \\
K_{\mathrm{HC}} \delta x_{s}{ }^{n}, & \delta x_{s} \geq 0 \wedge \dot{x}_{s}<0 \\
0, & \text { else. }\end{cases}
\end{aligned}
$$

In Eqs. (5) and (6), $K$ and $B$ denote stiffness and damping coefficients, $n$ is a constant, and $\delta x_{s}$ represents the environment compression.

The selection of the proper model between $\mathrm{KV}$ and $\mathrm{HC}$ for the environment is carried out by the hybrid object modeling approach, which is proposed in [15] and augmented in [16]. This approach is represented by operator $S$ as follows:

$$
S\left(K_{\mathrm{KV}}, t\right)= \begin{cases}\mathrm{KV}, & K_{\mathrm{KV}} \geq K_{t h} \vee \delta t \leq t_{t h} \\ \mathrm{HC}, & \text { else, }\end{cases}
$$

where $K_{t h}$ and $t_{t h}$ are stiffness and time thresholds, respectively, and $\delta t$ is defined as follows:

$$
\delta t \triangleq t-t_{w}
$$

where $t_{w}$ specifies the time of the first interaction between the slave robot and the environment.

\section{Estimation of environment parameters}

As mentioned in Section 1, in model-mediated teleoperation, the environment is virtually created on the master side. Therefore, a particular approach should be adopted for this purpose from different methods proposed in the literature. Relevant approaches can be divided into two main categories of model-based and model-free. An example of a model-free approach is [17] in which the environment force is predicted by a neural network on the slave side and is reproduced on the master side. However, our approach in this paper is model-based. Thus, an algorithm first estimates the environment parameters ( $K_{\mathrm{KV}}$ and $B_{\mathrm{KV}}$ for $\mathrm{KV}$ and $K_{\mathrm{HC}} ; B_{\mathrm{HC}}$ and $n$ for $\mathrm{HC}$ ) on the slave side, and then the estimated parameters are sent to the master side in order to create the virtual environment.

Several methods exist for determining the parameters of a system. There are numerical approaches, such as [18], which are mainly proposed to identify parameters of relatively complicated mechanical systems. Nonetheless, based on the dynamics of the environments defined by Eqs. (5) and (6), a simpler algorithm can be employed for the sake of parameter estimation in this paper. Hence, the SPRLS [19], which is a simple, yet powerful, recursive algorithm, seems a suitable choice. According to this algorithm, the actual and estimated systems should be linearized in the following form:

$$
\begin{aligned}
& y=\theta^{T} \Phi, \\
& \hat{y}=\hat{\theta}^{T} \Phi,
\end{aligned}
$$

where $y$ and $\hat{y}$ are the actual and estimated outputs, and $\theta$ and $\hat{\theta}$ are actual and estimated parameters, respectively, and $\Phi$ is the vector of inputs.

If the estimation error is defined as $\hat{e} \triangleq y-\hat{y}$, then vector $\hat{\theta}$ can be obtained from the following recursive relations:

$$
\begin{aligned}
& L_{i}=P_{i-1} \Phi_{i}\left(1+\Phi_{i}^{T} P_{i-1} \Phi_{i}\right)^{-1}, \\
& P_{i}=\left(I-L_{i} \Phi_{i}^{T}\right) P_{i-1}+\beta N I N T\left(\gamma \hat{e}_{i-1}^{2}\right) I, \\
& \hat{\theta}_{i}=\hat{\theta}_{i-1}+L_{i}\left(y_{i}-\Phi_{i}^{T} \hat{\theta}_{i-1}\right),
\end{aligned}
$$

where $\beta$ and $\gamma$ are constants, $I$ is the identity matrix, and $i=1,2, \cdots, N I N T($.$) operator is also defined as$ follows: 


$$
N I N T(x) \triangleq \begin{cases}x, & x \geq 0.5 \\ 0, & 0 \leq x<0.5 .\end{cases}
$$

The next step is to adapt Eqs. (9) and (10) to the KV and $\mathrm{HC}$ models. For KV, this can be done conveniently due to its inherent linearity; therefore, we have:

$$
\begin{aligned}
& y_{\mathrm{KV}}=F_{e}, \\
& \Phi_{\mathrm{KV}}=\left[\begin{array}{ll}
\delta x & \dot{x}
\end{array}\right]^{T}, \\
& \theta_{\mathrm{KV}}=\left[\begin{array}{ll}
K_{\mathrm{KV}} & B_{\mathrm{KV}}
\end{array}\right]^{T} .
\end{aligned}
$$

However, adaptation to $\mathrm{HC}$ model is not attained as easily as KV. In [20] and [21], double-stage and singlestage methods are proposed, respectively, in order to identify linearized $\mathrm{HC}$ parameters to be used in the form of Eqs. (9) and (10). However, Schindeler and Hashtrudi-Zaad utilized a novel and robust procedure for linearizing the $\mathrm{HC}$ model [22]. In this method, which is called polynomial linearization, the term $\delta x_{s}^{n}$ in the $\mathrm{HC}$ model is expanded based on Taylor series around its center point of working space, thus yielding the following:

$$
(\delta x)^{n} \approx \nu_{0}+\nu_{1} \delta x+\nu_{2}(\delta x)^{2},
$$

where $\nu_{0}, \nu_{1}$, and $\nu_{2}$ are coefficients that are obtained by arranging the Taylor expansion according to Eq. (14). Now, the HC model can be rewritten as follows:

$F_{e}=\left\{\begin{array}{l}K_{\mathrm{HC}}\left(\nu_{1} \delta x_{s}+\nu_{2}\left(\delta x_{s}\right)^{2}\right)+B_{\mathrm{HC}}\left(\nu_{1} \delta x_{s}+\nu_{2}\left(\delta x_{s}\right)^{2}\right) \dot{x}_{s}, \\ \quad \delta x_{s} \geq 0 \wedge \dot{x}_{s} \geq 0 \\ K_{\mathrm{HC}}\left(\nu_{1} \delta x_{s}+\nu_{2}\left(\delta x_{s}\right)^{2}\right), \quad \delta x_{s} \geq 0 \wedge \dot{x}_{s}<0 \\ 0, \quad \text { else }\end{array}\right.$

where $\nu_{0}=0$ is assumed. Consequently, the linearized relations for the $\mathrm{HC}$ model are obtained as in Eq. (16).

$$
\begin{aligned}
y_{\mathrm{HC}} & =F_{e}, \\
\Phi_{\mathrm{HC}} & =\left[\begin{array}{llll}
(\delta x)^{2} & \delta x & (\delta x)^{2} \dot{x} & (\delta x) \dot{x}
\end{array}\right]^{T}, \\
\theta_{\mathrm{HC}} & =\left[\begin{array}{llll}
K_{\mathrm{HC}} \nu_{2} & K_{\mathrm{HC}} \nu_{1} & B_{\mathrm{HC}} \nu_{2} & B_{\mathrm{HC}} \nu_{1}
\end{array}\right]^{T} .
\end{aligned}
$$

\section{Control approach and stability analysis}

\subsection{Controller design for steady state}

The term steady state is adopted from [8] which implies the time when the slave robot is not experiencing new contact with an environment. In other words, the slave robot either is in free motion or has previously encountered a new environment and is now just maintaining the contact. In this situation, regular control

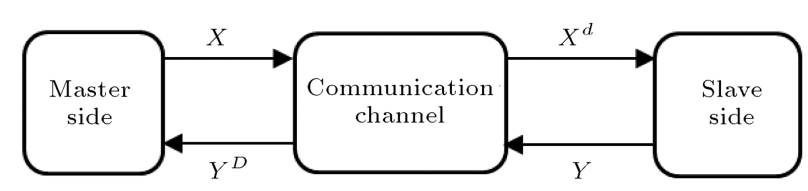

Figure 2. A scheme of the communication channel in the proposed teleoperation system. $X$ and $Y$ represent two arbitrary parameters.

approaches suffice. Although any suitably designed controller can be used, in this paper, impedance control and sliding mode control are implemented for the master and slave sides, respectively. Impedance control has the property of rendering the desired impedance on the master side, while the slave is able to track asymptotically the position of the master robot with a sliding mode controller.

Before continuing with design of controllers, it should be noticed that, in this paper, the delayed signals between the master and slave sides are designated by superscripts $d$ and $D$, as illustrated in Figure 2 and by the following relations:

$$
\begin{aligned}
& X\left(t-\tau_{m}\right) \triangleq X^{d}(t), \\
& Y\left(t-\tau_{s}\right) \triangleq Y^{D}(t),
\end{aligned}
$$

where $\tau_{m}$ and $\tau_{s}$ are communication time delays from master to slave side and slave to master side, respectively, and $X$ and $Y$ are two arbitrary parameters.

For impedance control of master side, a reference impedance behavior should be first defined [23,24]. Therefore, we have:

$$
M \ddot{x}_{m}+B \dot{x}_{m}+K x_{m}=F_{h}-\hat{F}_{e, m}
$$

where $F_{h}$ is the human or operator force and:

$$
\hat{F}_{e, m}= \begin{cases}\hat{\theta}_{s, \mathrm{KV}}^{D}{ }^{T} \Phi_{m, \mathrm{KV}}, & S\left(K_{\mathrm{KV}}, t\right)=\mathrm{KV} \\ \hat{\theta}_{s, \mathrm{HC}}^{D}{ }^{T} \Phi_{m, \mathrm{HC}}, & S\left(K_{\mathrm{KV}}, t\right)=\mathrm{HC}\end{cases}
$$

where subscripts $m$ and $s$ imply that the parameter should be inserted in or calculated on master and slave sides, respectively. $M, B$, and $K$ are the reference mass, damping coefficient, and stiffness, respectively. In addition, $\hat{F}_{(e, m)}$ represents the locally recreated environment force on the master side.

The term $\Phi_{m, \mathrm{HC}}$ in Eq. (20) includes the term $\delta x_{m}$, as can be regarded in Eq. (16b), which should be obtained from Eq. (21):

$$
\delta x_{m} \triangleq x_{m}-x_{w}^{D}
$$

where $x_{w}$ is the virtual environment position calculated on the slave side (see Section 4.2).

Finally, by combining Eqs. (3) and (19), the control input for the master side is attained: 


$$
\begin{aligned}
U_{m, s t}= & \left(b_{t, m}-\frac{m_{m}}{M} B\right) \dot{x}_{m}+\left(\frac{m_{m}}{M}-1\right) F_{h} \\
& -\frac{m_{m}}{M} \hat{F}_{e, m}+\left(k_{t, m}-\frac{m_{m}}{M} K\right) x_{m},
\end{aligned}
$$

where subscript st refers to the steady state.

For sliding mode control of the slave side, the error should be defined first. Here, the definition $e_{s t} \triangleq x_{s}-$ $x_{m}^{d}$ is used [25]. Then, the sliding surface is obtained in the following form:

$$
s_{s t}(x, t) \triangleq \dot{e}_{s t}+\lambda_{s t} e_{s t},
$$

where $\lambda_{s t}$ is strictly positive. Ultimately, the slave control signal can be derived by implementing the approach explained in the appendix:

$$
\begin{aligned}
U_{s, s t}= & \hat{b}_{s} \dot{x}_{s}+\hat{k}_{s} x_{s}+\hat{F}_{e} \\
& +\hat{m}_{s}\left(\ddot{x}_{m}^{d}-k_{\mathrm{gain}_{\mathrm{st}}} \operatorname{sat}\left(\frac{s_{s t}}{\varphi_{\mathrm{st}}}\right)-\lambda_{s t} \dot{e}_{s t}\right),
\end{aligned}
$$

where $\varphi$ is boundary layer width for reducing the chattering phenomenon, sat(.) is the saturation function, and the accent $\wedge$ refers to the uncertain value of the corresponding parameter.

Explanation of parameter $k_{\text {gain }_{s t}}$ can be found in the appendix. Furthermore, if one cannot or do not desire to use $\ddot{x}_{m}^{d}$ in Eq. (24) directly, he/she can replace it with the following relation, which is the delayed rearranged form of Eq. (19):

$$
\ddot{x}_{m}^{d}=\frac{1}{M}\left(F_{h}^{d}-\hat{F}_{e, m}^{d}-B \dot{x}_{m}^{d}-K x_{m}^{d}\right) .
$$

\subsection{Locating virtual environment on the master side}

In teleoperation systems, which are designed, based on model-mediated approach, locating the virtual environment on the correct position on the master side can greatly influence the transparency of the system. In fact, if the virtual environment is wrongly placed, $\delta x_{m}$ in Eq. (21) and, subsequently, vector $\Phi_{m}$ in Eq. (20) will deviate from their real value; thus, the calculated force on the master side will not follow the actual force on the slave side.

Until now, different researches have been conducted in line with this aim. One of the first approaches is based on [26], assuming the position of the virtual environment as one of the environment parameters (as in Eqs. 13(c) and 16(c)) and estimating it by an estimation algorithm. This approach requires the environment model to be as simple as possible, which is not always the case. More recent researches have also proposed the use of additional sensors, such as vision or proximity sensors, on the salve side for prior detection of the environment, such as the work done in [9].

However, the authors have previously proposed a method that is able to define the location of the virtual environment and the time of collision with environment on the slave side. This method, which is expressed in the following form, leads to the maximum possible transparency [16]:

$$
X_{w, j}=\left\{\begin{array}{l}
{\left[\begin{array}{ll}
x_{s, j-1} & t_{j-1}
\end{array}\right]^{T}, \quad\left|F_{e, j}\right|>F_{t h} \wedge\left|F_{e, j-1}\right| \leq F_{t h}} \\
X_{w, j-1}, \quad\left|F_{e, j}\right|>F_{t h} \wedge\left|F_{e, j-1}\right|>F_{t h} \\
{\left[\begin{array}{ll}
\Lambda & t_{j}
\end{array}\right]^{T}, \quad \text { else }}
\end{array}\right.
$$

where $X_{w} \triangleq\left[\begin{array}{ll}x_{w} & t_{w}\end{array}\right]^{T}$ (where $t_{w}$ is the time of the first interaction with the actual environment), subscripts $j$ and $j-1$ are the current and past time steps $(j=$ $1,2, \cdots)$, respectively, $F_{t h}$ is a force threshold, and $\Lambda$ is constant which is selected out of the working space of the robots. In this paper, $F_{t h}=0$ is considered.

Even though this method works well for relatively soft environments, it leads to model jump in interaction with hard environments [16]. Model jump is a phenomenon that produces a relatively high amount of force in the system in a limited time, thereby making the initial moments of the interaction hazardous for a teleoperation system.

Researches, such as [9], have previously considered reducing the effect of the model jump in the system. However, the main challenge still exists; generally, model jump and system transparency adversely affect each other. Therefore, if we aim to decrease the model jump phenomenon to the most possible extent, a separate control approach should be provided to the initial moment of contact with a new environment, which is discussed in the following section.

\subsection{Controller design for transition state}

The term transition state is adopted from [8] that implies the initial time of interaction with a new environment. In this situation, as mentioned before, the system may experience large contact forces, which may be extremely detrimental to it. This is generally called model jump effect. To prevent the system from being influenced by this effect, a novel control approach is proposed to transition state in this section, which is based mainly upon decoupling master and slave sides.

Mitra and Niemeyer proposed a technique in [27] whereby the virtual environment is gradually moved toward the correct position using a sliding surface (such as Eq. (23)) when the first contact with environment occurs. In this approach, however, the master main controller is still active during transition state, which results in degradation of transparency. To compensate for this, master and slave sides need to be fully decoupled during transition state. This means that the controller, which is active during steady state, should be deactivated during transition state.

To the authors' knowledge, the idea of decoupling was first implemented on a teleoperation system by 


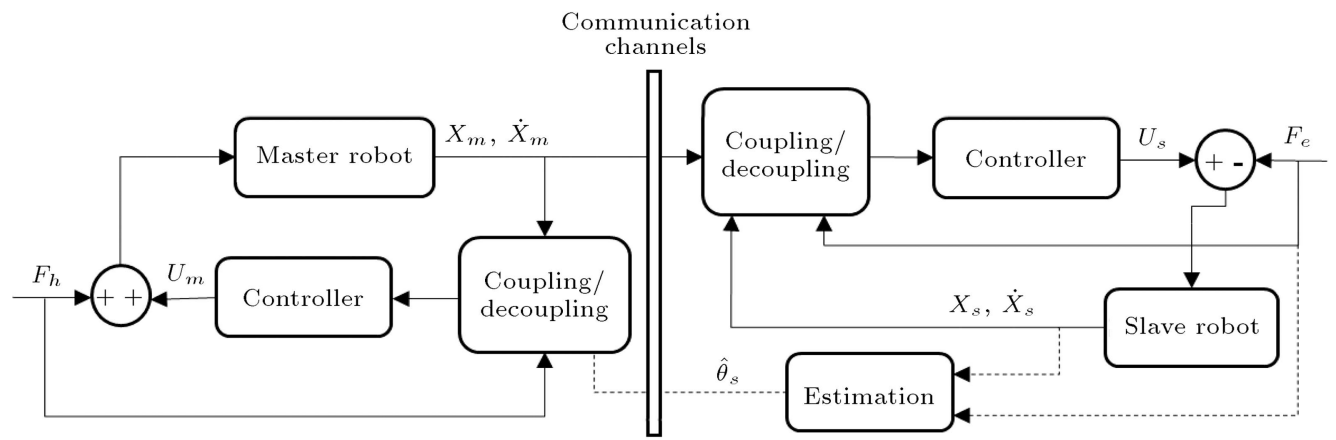

Figure 3. General scheme of the decoupling method. The coupling/decoupling blocks choose the input signals to be presented to the controller. Signals passing through the communication channels are only used when the coupling mode is active. Thus, if the decoupling mode is activated, the two control loops are closed separately and locally. $x, U, F_{e}, F_{h}$, and $\hat{\theta}_{s}$ represent position, control signal, environment force, human force, and estimated environment parameters, respectively. Subscripts $m$ and $s$ denote master and slave, respectively.

Smisek et al. [11]. However, the control strategy adopted there was different, and also only relatively stiff environments with the elastic model were considered, i.e., $F_{e}=K \delta x_{s}$. This paper extended this approach to a general case, which is independent of the environment model, guaranteeing system stability during transition state while maintaining high system transparency. A general scheme of the decoupling method is depicted in Figure 3.

Before designing controllers, a precise definition for transition state is presented. Transition state starts from the initial moment of interaction with a new environment and finishes at the time when both sides have become stable or, in other words, have returned to their initial contact positions.

For controlling the system during transition state, sliding mode control is utilized for both master and slave sides. Firstly, the error and sliding surface should be defined. In Eq. (23), the error was considered such that slave robot tracked the delayed position of the master robot, whereas, herein, the error must be defined with the aim of reaching the location of the initial contact. Hence, the error definitions for master and slave sides are presented as follows:

$$
\begin{aligned}
& e_{s, t r} \triangleq x_{s}-x_{w}, \\
& e_{m, t r} \triangleq x_{m}-x_{w}^{D},
\end{aligned}
$$

where subscript $t r$ denotes the transition state, and subscripts $m$ and $s$ denote master and slave sides, respectively.

Secondly, the sliding surfaces are defined as follows:

$$
\begin{aligned}
& s_{s, t r}(x, t) \triangleq \dot{e}_{s, t r}+\lambda_{s, t r} e_{s, t r}, \\
& s_{m, t r}(x, t) \triangleq \dot{e}_{m, t r}+\lambda_{m, t r} e_{m, t r} .
\end{aligned}
$$

Finally, similar to the procedure for Eq. (24), the control signals for both sides in transition state can be achieved as mentioned in the appendix:

$$
\begin{aligned}
U_{s, t r}= & \hat{b}_{s} \dot{x}_{s}+\hat{k}_{s} x_{s}+\hat{F}_{e} \\
& +\hat{m}_{s}\left(-k_{\mathrm{gain}_{s, t r}} \operatorname{sat}\left(\frac{s_{s, t r}}{\varphi_{s, t r}}\right)-\lambda_{s, t r} \dot{e}_{s, t r}\right),(31) \\
U_{m, t r}= & \hat{b}_{t, m} \dot{x}_{m}+\hat{k}_{t, m} x_{m}-\hat{F}_{e, m} \\
& +\hat{m}_{m}\left(-k_{\text {gain }_{m, t r}} \operatorname{sat}\left(\frac{s_{m, t r}}{\varphi_{m, t r}}\right)-\lambda_{m, t r} \dot{e}_{m, t r}\right) .
\end{aligned}
$$

Note that $x_{w}$ and $x_{w}^{D}$ are constants by definition (as defined in Eq. (26)). Thus, taking the derivatives of the defined errors in Eqs. (27) and (28), we can easily conclude that $\dot{e}_{s, t r}=\dot{x}_{s}$ and $\dot{e}_{m, t r}=\dot{x}_{m}$.

Obviously, Eqs. (31) and (32) do not contain any of the environment parameters. Therefore, this control strategy can be incorporated in a teleoperation system that deals with not only the environments defined by Eqs. (5) and (6) (which can be indeed a good approximation for most environments), but also any other environment with different dynamics.

The important point which is considered in transition state is that the operator exerts no force during this period [11]. In other words, when the operator notices the first signal of collision between the slave robot and the environment, he/she ceases pushing the master robot forward while still completely grasping it. When the transition state has finished, the operator reinitializes the operation.

\subsection{Discussion of system stability}

Overall stability of a teleoperation system should be discussed through two aspects. One aspect concerns the stability of the controllers designed on master and slave sides, and the other is the stability of the 
communication channels. In this paper, the former aspect is fulfilled readily due to utilizing impedance and sliding mode controllers, which are well known to be stable if tuned suitably. The latter, however, requires a deeper analysis, since it deals with the delayed signals that are transmitted through communication channels.

Considering classical control architectures described in Section 1, generally, two well-known approaches are employed to analyze the stability of the communication channels in a teleoperation system, though other approaches exist, too. The first one is absolute stability, for which the necessary and sufficient conditions are provided by Llewellyn's stability criterion [28]. An example of a design based on absolute stability can be found in [3]. The second one is passivity, which is adopted to analyze the stability of the system from energy generation point of view. An example of a passivity-based design can be found in [29]. These methods, however, make efforts to stabilize a teleoperation system basically when merely delayed signals are available on each side of it, which is not the case when it comes to model-mediated teleoperation.

As explained before, in model-mediated teleoperation, the master side is in interaction with a local virtual environment instead of the delayed force signal from the slave side. Accordingly, the master control loop is closed locally and, considering passive human operator and environment, the system is always stable (the master local loop could be inferred from Figure 1, too). The only remaining stability issues are thus as follows. First, the estimation algorithm must be convergent, which is attained in this paper by using the SPRLS algorithm. Second, the transition state must be controlled so that it does not destabilize the system, which is addressed in this paper.

Finally, it should be noted that the aforementioned analysis pertains to the slave-to-master direction of the teleoperation system applied in this work. The stability analysis regarding the master-to-slave direction is analogous to that in the previous works in this field, e.g. [30]. In addition, note that some researches, such as [10], have recently extended the concept of passivity to the context of model-mediated teleoperation. Despite having the same name and overall concept, these works should be separated from the conventional passivity analysis mentioned in the beginning of this section due to the difference in their control architectures.

\section{Simulation results}

To study the validity of the proposed approach, simulation results are presented for the designed teleoperation system in this section.

For simulating human operator force, the following function is considered

$$
F_{h}=\left\{\begin{array}{l}
2 t, \quad 0 \leq t<2 \\
4, \quad 2 \leq t<T \wedge t \in \text { steady state } \\
0, \quad 2 \leq t<T \wedge t \in \text { transition state } \\
\frac{1}{7}(t-6)+4, \quad T \leq t<13 \\
\frac{5}{2}(t-15), \quad 13 \leq t \leq 15
\end{array}\right.
$$

where $T$ is the time when the transition state finishes and can be tuned based on the operation circumstances. Herein, we determined $T=6 \mathrm{~s}$ by trial and error. In addition, when $F_{h}$ is zero, it indicates that the human operator has stopped pushing forward the master robot and is waiting for the system to reach a stable point. Note that, in Eq. (33), $t$ is in seconds.

A time delay of $0.5 \mathrm{~s}$ for both communication channels is considered (equivalent to a round-trip time delay of $1 \mathrm{~s}$ ), which means that $\tau_{m}=\tau_{s}=0.5 \mathrm{~s}$. In addition, an uncertainty interval is assumed for the mass of the slave robot as follows:

$$
1.058 \mathrm{~kg} \leq m_{s} \leq 1.267 \mathrm{~kg}
$$

Furthermore, in order to validate the generality of the proposed approach, two sample hard and soft environments are considered for interaction with the slave robot. Although hard and soft are intrinsically qualitative terms, it is common in the literature to assume an environment with stiffness bigger than $2500 \mathrm{~N} / \mathrm{m}$ (i.e., $K>2500 \mathrm{~N} / \mathrm{m}$ ) as hard and, otherwise, as soft $[15,20]$. For damping coefficient, however, such a general convention does not exist; however, it seems a good estimation to consider the ratio $\frac{K}{B} \approx 30$ $\mathrm{s}^{-1}[15]$. It is also a suitable approximation to consider $1<n<2$ for exponent $n$ [20,22]. The properties of the simulated environments, in addition to other assumptions, for the system are mentioned in Tables 1 to 6 . The tuning of the sliding mode controllers (Tables

Table 1. Dynamical properties of the master and slave robots (Phantom Omni for master and Novint Falcon for slave) [24].

\begin{tabular}{ccc}
\hline Parameter & Value & Unit \\
\hline$m_{m}$ & 0.223 & $\mathrm{~kg}$ \\
$b_{m}$ & 17.227 & $\mathrm{Ns} / \mathrm{m}$ \\
$k_{m}$ & 6.286 & $\mathrm{~N} / \mathrm{m}$ \\
$M$ & 0.223 & $\mathrm{~kg}$ \\
$B$ & 20.827 & $\mathrm{Ns} / \mathrm{m}$ \\
$K$ & 40.286 & $\mathrm{~N} / \mathrm{m}$ \\
$m_{s}$ & 1.158 & $\mathrm{~kg}$ \\
$b_{s}$ & 115.40 & $\mathrm{Ns} / \mathrm{m}$ \\
$k_{s}$ & 31.46 & $\mathrm{~N} / \mathrm{m}$ \\
\hline
\end{tabular}


Table 2. Dynamical properties of the human hand [14].

\begin{tabular}{ccc}
\hline Parameter & Value & Unit \\
\hline$b_{a}$ & 3.6 & $\mathrm{Ns} / \mathrm{m}$ \\
$k_{a}$ & 40 & $\mathrm{~N} / \mathrm{m}$ \\
\hline
\end{tabular}

Table 3. Parameters of sliding mode controller for steady state.

\begin{tabular}{cc}
\hline Parameter & Value \\
\hline$\eta_{s t}$ & 15 \\
$\lambda_{s t}$ & 1 \\
$\varphi_{s t}$ & 0.1 \\
$K_{\text {gain }_{s t}}$ & $1.1 K_{\text {gain }_{\text {, min }}}$ \\
\hline
\end{tabular}

Table 4. Parameters of sliding mode controllers for transition state.

\begin{tabular}{|c|c|}
\hline Parameter & Value \\
\hline$\eta_{s, t r}$ & 15 \\
\hline$\lambda_{s, t r}$ & 1 \\
\hline$\varphi_{s, t r}$ & 0.3 \\
\hline $\mathbf{K}_{\text {gain }_{s, t r}}$ & $1.1 \mathrm{~K}_{\text {gain }, \min _{s, t r}}$ \\
\hline$\eta_{m, t r}$ & 15 \\
\hline$\lambda_{m, t r}$ & 1 \\
\hline$\varphi_{m, t r}$ & 0.3 \\
\hline $\mathbf{K}_{\text {gain }_{m, t r}}$ & $1.1 \mathrm{~K}_{\text {gain, } \min _{m, t r}}$ \\
\hline
\end{tabular}

Table 5. Parameters of the hard environment, the corresponding SPRLS algorithm, and the hybrid object modeling approach.

\begin{tabular}{ccc}
\hline Parameter & Value & Unit \\
\hline$K_{\mathrm{KV}}$ & 6000 & $\mathrm{~N} / \mathrm{m}$ \\
$B_{\mathrm{KV}}$ & 230 & $\mathrm{Ns} / \mathrm{m}$ \\
$\lambda$ & 0.1 & - \\
$\beta$ & 30000 & - \\
$\hat{\boldsymbol{\theta}}_{\mathbf{0}}$ & {$[3000100]^{T}$} & - \\
$P_{0}$ & $I_{2}{ }^{\mathrm{a}}$ & - \\
$\hat{e}_{0}$ & 3 & - \\
SPRLS sample time & 0.0005 & $\mathrm{~s}$ \\
$K_{t h}$ & 2500 & $\mathrm{~N} / \mathrm{m}$ \\
$t_{t h}$ & 0.1 & $\mathrm{~s}$ \\
\hline
\end{tabular}

${ }^{a} I$ is the identity matrix.

Table 6. Parameters of the soft environment, the corresponding SPRLS algorithm, and the hybrid object modeling approach.

\begin{tabular}{ccc}
\hline Parameter & Value & Unit \\
\hline$K_{\mathrm{HC}}$ & 1000 & $\mathrm{~N} / \mathrm{m}$ \\
$B_{\mathrm{HC}}$ & 30 & $\mathrm{Ns} / \mathrm{m}$ \\
$n$ & 1.5 & - \\
$\gamma$ & 19000 & - \\
$\beta$ & 1 & - \\
$\hat{\boldsymbol{\theta}}_{\mathbf{0}}$ & {$[4004001313]^{T}$} & - \\
$P_{0}$ & $I_{4}{ }^{\mathrm{a}}$ & - \\
$\hat{e}_{0}$ & 2 & - \\
SPRLS sample time & 0.0005 & $\mathrm{~s}$ \\
$K_{t h}$ & 2500 & $\mathrm{~N} / \mathrm{m}$ \\
$t_{t h}$ & 0.1 & $\mathrm{~s}$ \\
\hline
\end{tabular}

\footnotetext{
${ }^{a} I$ is the identity matrix.
}

3 and 4) and the parameters of the SPRLS algorithm (Tables 5 and 6 ) was conducted manually.

The results of the simulation are also illustrated in Figures 4 to 11. From Figures 4 and 8, it can be seen that when a new environment is encountered, whether hard or soft, the transition state starts and continues until both master and slave sides become

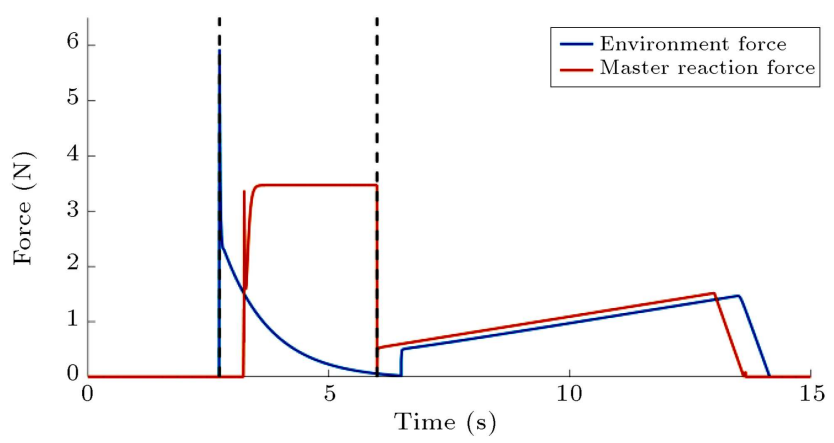

Figure 4. Environment and master reaction forces in interaction with the hard environment. The black dashed lines represent the start and end of the transition state.

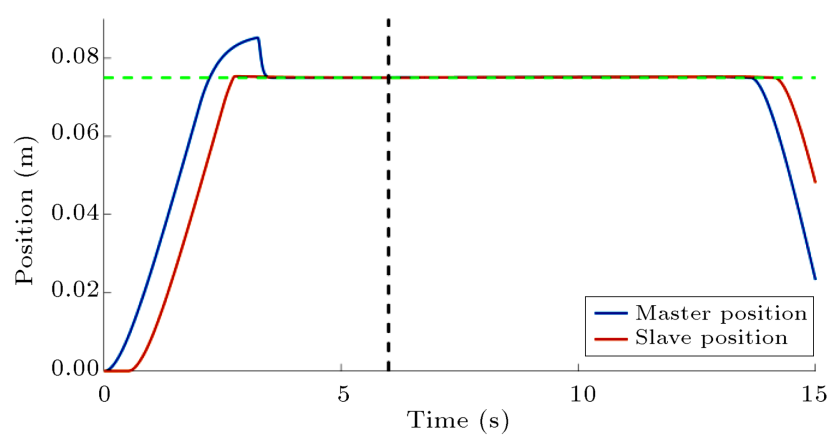

Figure 5. Master and slave positions in interaction with the hard environment. The black dashed line (vertical) represents the end of the transition state (the transient state starts when the slave reaches the environment for the first time). The green dashed line (horizontal) shows the position of the environment on the slave side.

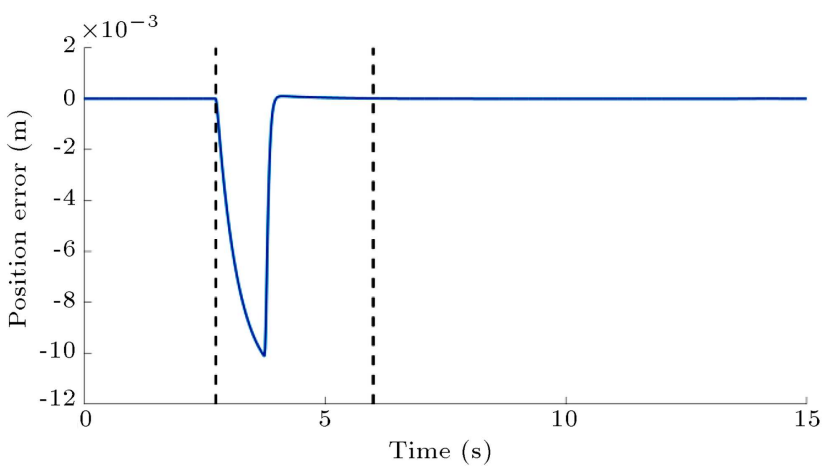

Figure 6. Position error in interaction with the hard environment. The black dashed lines represent the start and end of the transition state. Although otherwise defined in Section 4.3, the error depicted for the transition state is based on the definition in Section 4.1. 


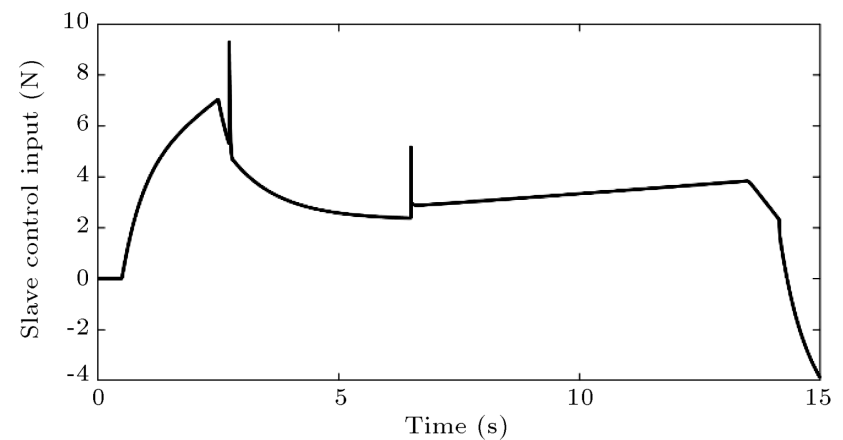

Figure 7. Slave control input in interaction with the hard environment.

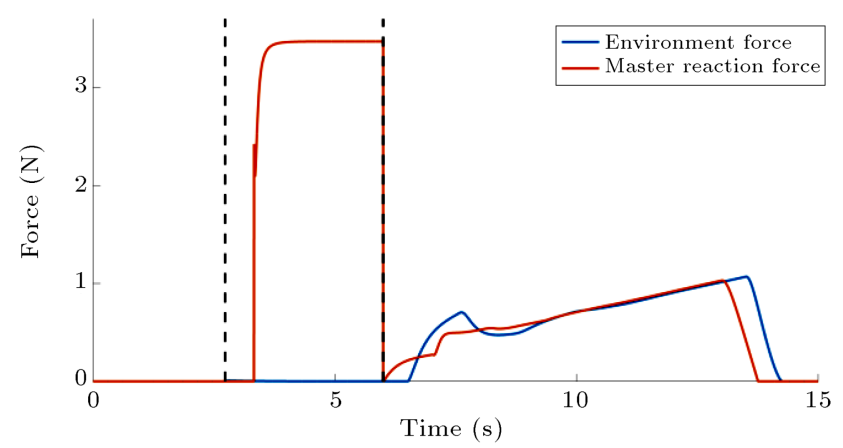

Figure 8. Environment and master reaction forces in interaction with the soft environment. The black dashed lines represent the start and end of the transition state.

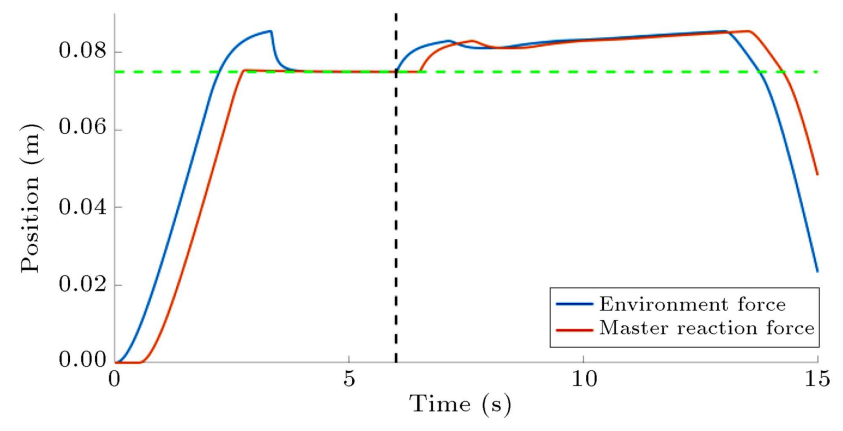

Figure 9. Master and slave positions in interaction with the soft environment. The black dashed line (vertical) represents the end of the transition state (the transient state starts when the slave reaches the environment for the first time). The green dashed line (horizontal) shows the position of the environment on the slave side.

stable. After that, the system goes to the steady state again while normal operation is being carried out. Two important points can be inferred from these two figures: Firstly, the master robot is able to track the environment force ahead accurately, due to the predictive architecture used, during the steady state (which shows high transparency); secondly, the model jump effect has been drastically decreased, especially if compared with the results presented in [16] (showing increased stability).

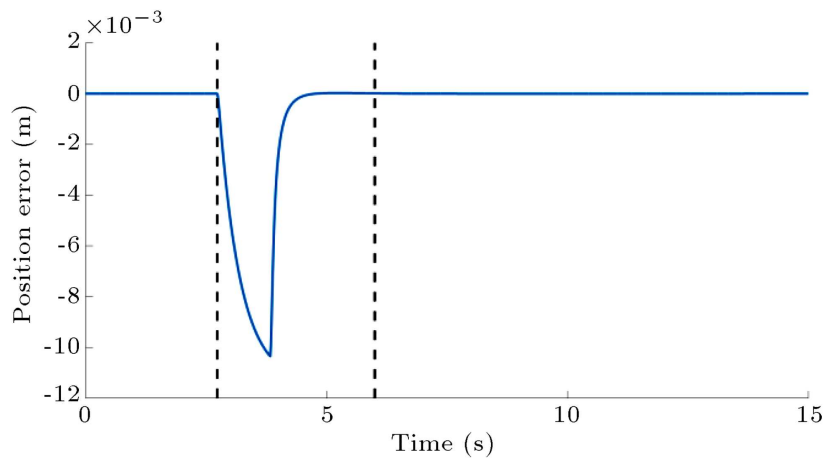

Figure 10. Position error in interaction with the soft environment. The black dashed lines represent the start and end of the transition state. Although otherwise defined in Section 4.3, the error depicted for the transition state is based on the definition in Section 4.1.

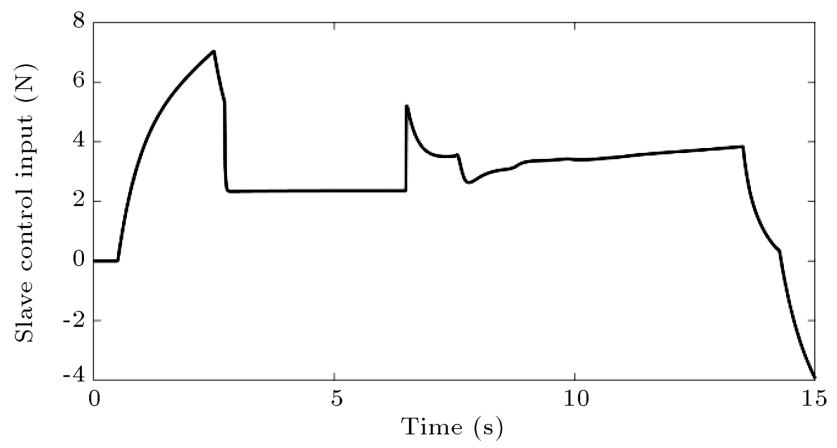

Figure 11. Slave control input in interaction with the soft environment.

Figures 5 and 9 show that, for both hard and soft environments, the slave robot tracks the position of the master robot behind (due to the inherent time delay in the communication channels). In Figures 6 and 10, the position error between the master and slave robots is depicted that further proves the quality of the position tacking of the system for both hard and soft contacts and during free motion (no contact with environment). Finally, the control input of the slave robot is illustrated in Figures 7 and 11 during contact with hard and soft environments, respectively. It should be pointed out that the master control input is equal to the master reaction force (in magnitude) as shown in Figures 4 and 8 . Moreover, note that transparency cannot be considered for transition state due to its nature. To put it simply, transparency can be defined when signals are being transmitted between two sides of a teleoperation system, while no signal is transmitted during transition state by definition.

Despite high transparency, it can be observed that, after the end of the transition state during interaction with the soft environment (Figure 8), transparency is not as high as that when it is in interaction with the hard environment for some time. The reason is that the system has not yet decided which model, 
i.e., $\mathrm{KV}$ or $\mathrm{HC}$, to use for the virtual environment. Considering hybrid object modeling approach defined by Eq. (7), it can be concluded that when encountering a soft environment, the system needs a particular amount of time to change the default model of virtual environment (from $\mathrm{KV}$ to $\mathrm{HC}$ ) on the master side. Considering the time delays in the communication channels, the system carries out this task in approximately $\tau_{m}+\tau_{s}+t_{t h}$ seconds. This effect is inevitable during the first interaction with a completely unknown environment. However, in subsequent contacts, this phenomenon can be avoided by reproducing the first contact's data if the environment is known to remain unchanged during the operation.

Another issue that may arise when utilizing the proposed full decoupling method is the time when the operator is required to wait until the system becomes stable during the transition state. The user might conceive of this waiting duration as a distracting factor. This interval, however, could be adjusted based on the desired application by suitably tuning the sliding mode controller parameters, although it cannot be completely omitted.

\section{Conclusions and future work}

In this paper, the idea of decoupling master and slave sides in a teleoperation system during a new contact with an environment for decreasing the model jump effect was addressed. A novel position control approach based on sliding mode control was proposed for transition state, while the master and slave sides were fully decoupled. The principal advantage of the proposed approach, in comparison with previous works, is its independence of environment model. This means that, regardless of environment type and system dynamics, the proposed control approach leads the system towards stability when it first encounters a new unknown environment, while high transparency is preserved.

In addition, this approach is capable of dealing with any finite time delay in communication channels. Thus, with suitable tuning of different parameters of controllers, the stability of teleoperation system is guaranteed in the presence of large amounts of time delay.

Despite guaranteed stability and high transparency, the waiting time interval during transition state, in which the operator must stop the operation until the system reaches stability, can be of inconvenience for the user. Nevertheless, suitably tuning the relevant sliding mode controller parameters can partly compensate for this issue. Interaction with an unknown soft environment for the first time may also provide an inconvenient condition for the user, as thoroughly discussed in Section 5. These two sources of inconvenience are to be further investigated through experimental tests in future works. How and how much these two sources can affect the haptic feeling of the user and how to mitigate them, if necessary, can be discussed.

Moreover, this paper used the predictive control approach merely for slave-to-master direction. In order to further augment the transparency and stability of the system, master-to-slave state prediction can also be taken into account in the presence of environment force in future works.

\section{References}

1. Aliaga, I., Rubio, A., and Sanchez, E. "Experimental quantitative comparison of different control architectures for master-slave teleoperation", IEEE Transactions on Control Systems Technology, 12(1), pp. 2-11 (2004).

2. Hua, C.-C., Yang, Y., and Guan, X. "Neural networkbased adaptive position tracking control for bilateral teleoperation under constant time delay", Neurocomputing, 113, pp. 204-212 (2013).

3. García-Valdovinos, L.-G., Parra-Vega, V., and Arteaga, M.A. "Observer-based sliding mode impedance control of bilateral teleoperation under constant unknown time delay", Robotics and Autonomous Systems, 55(8), pp. 609-617 (2007).

4. Cho, H.C. and Park, J.H. "Impedance control with variable damping for bilateral teleoperation under time delay", JSME International Journal Series C Mechanical Systems, Machine Elements and Manufacturing, 48(4), pp. 695-703 (2005).

5. Weber, C., Nitsch, V., Unterhinninghofen, U., Farber, B., and Buss, M. "Position and force augmentation in a telepresence system and their effects on perceived realism", Third Joint Eurohaptics Conference and Symposium on Haptic Interfaces for Virtual Environment and Teleoperator Systems, Salt Lake City, UT, USA, pp. 226-231 (2009).

6. Hannaford, B. "A design framework for teleoperators with kinesthetic feedback", IEEE Transactions on Robotics and Automation, 5(4), pp. 426-434 (1989).

7. Uddin, R. and Ryu, J. "Predictive control approaches for bilateral teleoperation", Annual Reviews in Control, 42, pp. 82-99 (2016).

8. Xu, X., Cizmeci, B., Schuwerk, C., and Steinbach, E. "Model-mediated teleoperation: Toward stable and transparent teleoperation systems", IEEE Access, 4, pp. 425-449 (2016).

9. Xu, X., Paggetti, G., and Steinbach, E. "Dynamic model displacement for model-mediated teleoperation", IEEE World Haptics Conference (WHC), Daejeon, Korea, pp. 313-318 (2013).

10. Xu, X., Schuwerk, C., and Steinbach, E. "Passivitybased model updating for Model-mediated Teleoperation", IEEE International Conference on Multimedia 
\& Expo Workshops (ICMEW), Turin, Italy, pp. 1-6 (2015).

11. Smisek, J., van Paassen, R.M., and Schiele, A. "Naturally-transitioning rate-to-force controller robust to time delay by model-mediated teleoperation", IEEE International Conference on Systems, Man, and Cybernetics (SMC), Hong Kong, pp. 3066-3071 (2015).

12. Willaert, B., Van Brussel, H., and Niemeyer, G. "Stability of model-mediated teleoperation: discussion and experiments", International Conference on Human Haptic Sensing and Touch Enabled Computer Applications, Tampere, Finland, pp. 625-636 (2012).

13. Lawrence, D.A. "Stability and transparency in bilateral teleoperation", IEEE Transactions on Robotics and Automation, 9(5), pp. 624-637 (1993).

14. Speich, J.E., Shao, L., and Goldfarb, M. "Modeling the human hand as it interacts with a telemanipulation system", Mechatronics, 15(9), pp. 1127-1142 (2005).

15. Achhammer, A., Weber, C., Peer, A., and Buss, M. "Improvement of model-mediated teleoperation using a new hybrid environment estimation technique", IEEE International Conference on Robotics and Automation (ICRA), Anchorage, Alaska, USA, pp. 53585363 (2010).

16. Yazdankhoo, B. and Beigzadeh, B. "Improving transparency in bilateral teleoperation systems based on model-mediated approach", Modares Mechanical Engineering, 17(1), pp. 273-283 (2017) (in Persian).

17. Smith, A.C. and Hashtrudi-Zaad, K. "Neural networkbased teleoperation using Smith predictors", IEEE International Conference Mechatronics and Automation, Niagara Falls, Canada, 3, pp. 1654-1659 (2005).

18. Tarvirdizadeh, B., Khanmirza, E., Ebrahimi, M., Kalhor, A., and Vakilipour, S. "An efficient numerical and experimental system identification approach for a flexible manipulator", Engineering Computations, 32(8), pp. 2467-2490 (2015).

19. Park, D.-J. and Jun, B.-E. "Selfperturbing recursive least squares algorithm with fast tracking capability", Electronics Letters, 28(6), pp. 558-559 (1992).

20. Diolaiti, N., Melchiorri, C., and Stramigioli, S. "Contact impedance estimation for robotic systems", IEEE Transactions on Robotics, 21(5), pp. 925-935 (2005).

21. Haddadi, A. and Hashtrudi-Zaad, K. "Real-time identification of Hunt-Crossley dynamic models of contact environments", IEEE Transactions on robotics, $\mathbf{2 8}(3)$, pp. 555-566 (2012).

22. Schindeler, R. and Hashtrudi-Zaad, K. "Polynomial linearization for real-time identification of environment Hunt-Crossley models", IEEE Haptics Symposium (HAPTICS), Pennsylvania, USA, pp. 173-178 (2016).

23. Sadeghi, M.S., Momeni, H.R., and Amirifar, R. "h $\infty$ and 11 control of a teleoperation system via LMIs", Applied Mathematics and Computation, 206(2), pp. 669-677 (2008).
24. Hilliard, T. and Pan, Y.-J. "Stabilization of asymmetric bilateral teleoperation systems for haptic devices with time-varying delays", American Control Conference, Washington, DC, USA, pp. 4538-4543 (2013).

25. Park, J.H. and Cho, H.C. "Sliding mode control of bilateral teleoperation systems with force-reflection on the internet", IEEE/RSJ International Conference on Intelligent Robots and Systems (IROS), Takamatsu, Japan, 2 pp. 1187-1192 (2000).

26. Tzafestas, C., Velanas, S., and Fakiridis, G. "Adaptive impedance control in haptic teleoperation to improve transparency under time-delay", IEEE International Conference on Robotics and Automation (ICRA), Pasadena, CA, USA, pp. 212-219 (2008).

27. Mitra, P. and Niemeyer, G. "Model-mediated telemanipulation", The International Journal of Robotics Research, 27(2), pp. 253-262 (2008).

28. Llewellyn, F. "Some fundamental properties of transmission systems", Proceedings of the IRE, 40(3), pp. 271-283 (1952).

29. Lee, D. and Spong, M.W. "Passive bilateral teleoperation with constant time delay", IEEE Transactions on Robotics, 22(2), pp. 269-281 (2006).

30. Cho, H.C., Park, J.H., Kim, K., and Park, J.-O. "Sliding-mode-based impedance controller for bilateral teleoperation under varying time-delay", IEEE International Conference on Robotics and Automation (ICRA), Seoul, Korea, 1 pp. 1025-1030 (2001).

\section{Appendix}

The procedure for determining the control input of the sliding mode control for the slave side in steady state is explained here. First, Eq. (4) is rewritten in the following form:

$$
\ddot{x}_{s}=f\left(\mathbf{x}_{\mathbf{s}}, t\right)+g\left(\mathbf{x}_{\mathbf{s}}, t\right) U_{s, s t},
$$

where $\mathbf{x}_{\mathbf{s}}=\left[\begin{array}{ll}\mathbf{x}_{s} & \dot{\mathbf{x}_{s}}\end{array}\right]^{T}$ is the state vector. In addition, $f \triangleq f\left(\mathbf{x}_{\mathbf{s}}, t\right)$ and $g \triangleq g\left(\mathbf{x}_{\mathbf{s}}, t\right)$ can be obtained as follows:

$$
\begin{aligned}
& f=\frac{1}{m_{s}}\left(-b_{s} \dot{x}_{s}-k_{s} x_{s}-F_{e}\right), \\
& g=\frac{1}{m_{s}} .
\end{aligned}
$$

Herein, $f$ and $g$ can have uncertainties, but in known ranges which are defined as follows:

$$
\begin{aligned}
& |\hat{f}-f| \leq F, \\
& \frac{1}{\alpha} \leq \frac{g}{\hat{g}} \leq \alpha,
\end{aligned}
$$

where $\hat{f}$ and $\hat{g}$ represent the estimated values of $f$ and $g$, respectively. In Eq. (A.5), $\hat{g}$ and $\alpha$ are defined according to the following relations: 


$$
\begin{aligned}
& \hat{g} \triangleq \sqrt{g_{\max } g_{\min }} \\
& \alpha \triangleq \sqrt{\frac{g_{\max }}{g_{\min }}}
\end{aligned}
$$

where subscripts max and min denote the maximum and minimum values of the corresponding parameter, respectively. Next, by having the sliding surface as defined in Eq. (23) and applying the condition $s_{s t}=$ $\dot{s}_{s t}=0$, the estimated control input is defined as follows:

$$
\hat{U}_{s, s t}=\frac{1}{\hat{g}} \hat{u}
$$

where,

$$
\hat{u} \triangleq-\hat{f}+\ddot{x}_{m}^{d}-\lambda_{s t} \dot{e}_{s t} .
$$

Finally, the sliding condition must be applied according to Eq. (A.10):

$$
s_{s t} \dot{s}_{s t} \leq \eta_{s t}\left|s_{s t}\right|
$$

where $\eta_{s t}$ is strictly positive. It can be shown that a control input in the following form satisfies Condition in Eq. (A.10):

$$
U_{s, s t}=\hat{U}_{s, s t}-\frac{1}{\hat{g}} k_{\mathrm{gain}_{s t}} \operatorname{sat}\left(\frac{s_{s t}}{\varphi_{s t}}\right) .
$$

In Eq. (A.11), $k_{\text {gain }_{s t}}$ is defined such that:

$$
\begin{aligned}
& k_{\text {gain }_{\mathrm{st}}} \geq k_{\mathrm{gain}_{\text {anin }} \text { st }}, \\
& k_{\text {gain,min }_{s t}} \triangleq \alpha\left(F+\eta_{s t}\right)+(\alpha-1)|\hat{u}| .
\end{aligned}
$$

Other parameters in Eq. (A.11) have been defined in Section 4.1. Ultimately, by rewriting Eq. (A.11) according to Eqs. (A.2), (A.3), (A.8), and (A.9), the control input can be obtained which is represented by Eq. (24) in Section 4.1.

Notice that the control inputs for transition state (represented by Eqs. (31) and (32) in Section 4.3) can also be achieved through the aforementioned process. The only alterations are that subscript st should be replaced by $t r$ (denoting the transition state) and also subscript $s$ should be replaced by $m$ for obtaining $U_{m, t r}$.

\section{Biographies}

Behnam Yazdankhoo received the BSc degree in Mechanical Engineering from Amirkabir University of Technology, Tehran, Iran in 2015. He is currently MSc student in the School of Mechanical Engineering at Iran University of Science and Technology, Tehran, Iran. His main research interests are design and control of haptic teleoperation systems and humanoid robots.

Borhan Beigzadeh received his $\mathrm{PhD}$ degree in Mechanical Engineering from Sharif University of Technology in 2011, working on dynamic walking systems and studying their correlation with dynamic passive/active manipulation systems. He then joined Iran University of Science and Technology (IUST) in Tehran where he established Biomechatronics and Cognitive Engineering Research Laboratory, and since then is an Assistant Professor at the School of Mechanical Engineering. His research interests cover non-linear dynamics and control, robotics, biomechatronics, and cognitive engineering. 